\title{
Fatores nutricionais associados à reprodução da fêmea bovina
}

\author{
Roberto Sartori ${ }^{1}$, Monique Mendes Guardieiro ${ }^{1}$ \\ ${ }^{1}$ Escola Superior de Agricultura "Luiz de Queiroz" - ESALQ, Universidade de São Paulo, Piracicaba, SP, Brasil - 13418-900.
}

RESUMO - O conhecimento da importância da nutrição sobre a reprodução dos animais, por produtores e pesquisadores, vem de longa data. Entretanto, estudos contemporâneos têm trazido novas perspectivas e informações mais detalhadas desta relação, permitindo um ajuste fino nas manipulações de dietas com o propósito de aumentar a produção e a reprodução em ruminantes. Apesar disso, especialmente em vacas de elevada produção leiteira, ainda há sérios problemas de fertilidade de ordem multifatorial, mas com grande envolvimento nutricional. Por outro lado, adição de certos ingredientes à dieta, como gordura rica em ácidos graxos polinsaturados, e redução do fornecimento de proteína degradável no rúmen têm, em diversas ocasiões, resultado em incremento nos índices reprodutivos em bovinos leiteiros e de corte. Nesse sentido, este artigo tem a proposta de apresentar e discutir resultados de alguns trabalhos relevantes sobre o tema, principalmente aqueles relacionados à influência da energia ou proteína sobre a reprodução.

Palavras-chave: bovino, energia, fertilidade, fisiologia, gordura, proteína

\section{Nutritional factors associated with reproduction in heifers and cows}

\begin{abstract}
The knowledge of the importance of nutrition on animal reproduction is well known for producers and researchers for a long time. However, contemporary studies have brought new perspectives and further details of this relationship, allowing fine-tuning of diets handling in order to increase production and reproduction in ruminants. Nevertheless, especially in high-producing dairy cows, there are still serious multifactorial fertility problems, with a heavy nutritional involvement. In contrast, addition of certain ingredients to the diet, such as fat rich in polyunsaturated fatty acids and reducing the supply of rumen degradable protein have on several occasions, resulted in an increased reproductive performance in dairy and beef cattle. Thus, this article presents and discusses results of some relevant papers on this subject, especially those related to the influence of energy or protein on reproduction.
\end{abstract}

Key Words: bovine, energy, fat, fertility, physiology, protein

\section{Introdução}

Dentre os fatores que exercem influência na reprodução, a nutrição tem um papel reconhecidamente importante por afetar diretamente aspectos da fisiologia e desempenho reprodutivo na fêmea bovina. Por exemplo, diversos estudos associaram a nutrição à queda da fertilidade, principalmente em vacas leiteiras e identificaram como causas potenciais o balanço energético negativo (BEN), evidenciado pela queda no escore de condição corporal (ECC) pós-parto (Moreira et al., 2000; Lopez-Gatius et al., 2002), os efeitos deletérios de dietas altamente energéticas (Wiltbank et al., 2006; Santos et al., 2008), os efeitos tóxicos de compostos nitrogenados (Butler, 1998; Dawuda et al., 2002; Rhoads et al., 2006), e as deficiências de vitaminas e/ou minerais (Ingraham et al., 1987; Arechiga et al., 1994, 1998). Além disso, ainda há muita controvérsia sobre os efeitos do "flushing” nutricional ou incremento na ingestão de matéria seca (IMS) sobre a fertilidade e produção embrionária em ruminantes (Diskin \& Morris 2008; Leroy et al., 2008a; Santos et al., 2008). Por outro lado, como aspecto positivo, diversos trabalhos têm demonstrado resultados favoráveis com o uso de suplementos à base de gordura sobre a função reprodutiva de fêmeas de corte e leite. Devido à grande quantidade de informações sobre o tema em questão e para evitar apresentá-las de forma superficial, a proposta deste artigo é discutir principalmente os estudos que focaram a influência da energia ou proteína na reprodução da fêmea bovina.

\section{Nutrição e condição corporal pós-parto}

O momento do retorno a ciclicidade após o parto em fêmeas bovinas é de fundamental importância para determinar quão cedo essas vacas vão conceber e consequentemente, definir o intervalo de partos. Esse 
retorno a ciclicidade depende de diversos fatores, tais como, raça, presença ou ausência de bezerro, produção leiteira, sanidade, número de parições, nutrição pré e pósparto e condição corporal ao parto e sua manutenção após o parto. Vacas de alta produção leiteira são as fêmeas geralmente acometidas por um BEN mais severo no período pós-parto. Durante as últimas semanas de gestação e início da lactação esses animais apresentam um período de BEN. O BEN ocorre, porque o pico de produção de leite se estabelece semanas antes do pico de IMS e a energia utilizada para manutenção e produção de leite é maior do que a energia adquirida pela alimentação.

Quando as vacas estão em BEN, as concentrações sanguíneas de ácidos graxos não esterificados (AGNEs), ureia e $\beta$-hidroxibutirato aumentam, enquanto as de IGF-I, glicose e insulina estão baixas, já que estes últimos são destinados à síntese de leite. Essa alteração nos níveis sanguíneos dessas substâncias está geralmente associada ao comprometimento da função ovariana e fertilidade. Leroy et al. (2005) detectaram concentrações elevadas de AGNEs no líquido folicular de vacas leiteiras em BEN poucos dias após o parto. Estudos in vitro demonstraram efeitos prejudiciais no desenvolvimento embrionário quando ovócitos foram maturados em um ambiente com altas concentrações de AGNEs e baixas concentrações de glicose (Leroy et al., 2008a). Da mesma forma, baixas concentrações de IGF-I também podem estar associadas a um desenvolvimento embrionário sub ótimo (Velazquez et al., 2005).

Segundo Beam \& Butler (1998), o atraso na ovulação pós-parto está diretamente associado ao estado energético da vaca, ou seja, quanto maior o BEN, maior o tempo para retorno a ciclicidade; bem como a efeitos adversos na eficiência reprodutiva subseqüente da vaca. Alguns pesquisadores sugerem que um retorno mais cedo a ciclicidade seja benéfico ao desempenho reprodutivo (Stevenson et al., 1983; Staples et al., 1990; Senatore et al., 1996; Darwash et al., 1997) devido ao aumento no número de ciclos estrais antes da inseminação artificial (IA) estar associado à maior taxa de concepção (TC) à primeira IA (Thatcher \& Wilcox, 1973). Por outro lado, quando vacas apresentam a primeira ovulação pós-parto muito cedo (<26 dias) há queda na eficiência reprodutiva e aumento na incidência de corpo lúteo (CL) persistente, que geralmente está associado à infecção e involução uterina retardada (Ball \& McEwan, 1998; Lamming \& Darwash, 1998; Smith \& Wallace, 1998; discutido por Sartori et al., 2004b).

Os efeitos deletérios do BEN após o parto podem ser exacerbados se, concomitantemente houver elevada perda de condição corporal nas vacas. Esta queda de condição corporal, muitas vezes independente do ECC que a vaca apresenta ao parto, está diretamente associada ao atraso na primeira ovulação e aumento nos dias para concepção (Wiltbank et al., 2006). Vacas com maior perda de ECC nas primeiras semanas de lactação apresentaram comprometimento da eficiência reprodutiva mais acentuado (Butler \& Smith, 1989; Ferguson, 1991), fato observado inclusive naquelas que pariram com ECC elevado (Zulu et al., 2002). Apesar de a avaliação do ECC ser uma ferramenta importante para o manejo nutricional e reprodutivo em bovinos, ela não pode ser utilizada sozinha. Por exemplo, em um estudo (Sartori et al., 2004a) que avaliou a ciclicidade em vacas de alta produção (44,5 kg de leite por dia) com 80 dias em lactação, em média, não se detectou diferença no ECC no grupo que apresentava ciclicidade normal comparado ao grupo com ciclicidade alterada $(\mathrm{ECC}=3,4$ vs 3,0, respectivamente; escala de 1 a 5 ).

Em bovinos de corte, o desempenho reprodutivo também está associado ao ECC (Bossis et al., 2000), o qual é um dos fatores que podem afetar o crescimento e persistência do folículo dominante (Rhodes et al., 1995) no período pósparto. Vacas de corte, geralmente têm um anestro pós-parto mais prolongado do que as de leite, devido, geralmente, à somatória de fatores como ECC baixo, nutrição inadequada e presença do bezerro (amamentação). Vacas primíparas são as mais acometidas por esses fatores por se encontrar em fase de crescimento. Para reduzir o anestro pós-parto, diferentes estratégias de manejo são indicadas e consistem em minimizar os efeitos negativos descritos acima como, por exemplo, realizar suplementação alimentar no período pós-parto, promover remoções temporárias estratégicas de bezerros ("shang”) ou esquemas de amamentação interrompida, ou mesmo lançar mão de tratamentos hormonais para indução de estro ou de ovulação (Williams, 1990; Baruselli et al., 2004; Sá Filho et al., 2009).

Tanto em vacas de corte quanto de leite, o mecanismo pelo qual o BEN, perda de condição corporal e amamentação relacionam-se ao atraso da ovulação pós-parto está geralmente associado à baixa pulsatilidade de hormônio luteinizante (LH; Butler \& Smith, 1989; Williams, 1990; Wiltbank et al., 2002). Como mencionado anteriormente, o BEN afeta níveis sistêmicos de IGF-I, insulina e glicose, os quais podem alterar a freqüência de pulsos de $\mathrm{LH}$, comprometendo, consequentemente, o crescimento folicular (Grimard et al., 1995). Em casos extremos, em períodos prolongados de subnutrição, as quantidades insuficientes de alimento na dieta influenciam o desenvolvimento folicular, chegando a ponto de causar condição anovulatória (Rhodes et al., 1996; Bossis et al., 2000; Wiltbank et al., 2002). O 
restabelecimento da pulsatilidade normal de LH é o fator determinante para o reinício do crescimento folicular e ciclicidade nas vacas pós-parto e uma das formas de acelerar esse processo é por meio do fornecimento de dietas adequadas durante os períodos pré e pós-parto.

Influência de dietas ricas em energia na fisiologia reprodutiva e fertilidade em bovinos

Fêmeas bovinas recebendo alta ingestão alimentar podem sofrer influência em diversos aspectos da fisiologia reprodutiva. Por exemplo, em dois estudos com novilhas de corte (Murphy et al., 1991; Mollo et al., 2007), os animais que foram subalimentados $(70 \%$ em relação à quantidade de mantença; 0,7M) apresentaram redução na persistência e no tamanho do folículo dominante em relação aos superalimentados (1,7 a 1,8M). Outros estudos descreveram alteração na população de folículos ao início de uma onda de crescimento folicular em novilhas ou vacas submetidas a diferentes níveis nutricionais. Da mesma forma, alta IMS/ energia está relacionada à queda na qualidade embrionária e consequentemente, na fertilidade de fêmeas ruminantes (Wiltbank et al., 2006; Sartori et al., 2007; Santos et al., 2008).

Dentre os fatores envolvidos nestes processos reprodutivos devem-se destacar a ação da glicose, IGF-I e insulina, cujas concentrações sangüíneas variam de acordo com o nível nutricional e estado fisiológico do animal (Webb et al., 2004). Como apresentado em artigos de revisão recentes (Sartori et al., 2007; Santos et al., 2008), alguns trabalhos detectaram efeitos positivos da alta ingestão alimentar associados a altas concentrações circulantes de IGF-I e insulina, na população folicular e número de ovulações; outros não detectaram efeito, mas a maioria detectou um efeito negativo. Experimentos que compararam fêmeas bovinas em categorias zootécnicas distintas, incluindo diferentes níveis de ingestão de alimento, também descreveram alterações na função ovariana e na fisiologia reprodutiva desses animais, a se destacar menores concentrações circulantes dos hormônios esteroides progesterona e estradiol - em fêmeas com maior ingestão alimentar, apesar de estas terem apresentado folículos e CLs maiores (revisado por Sartori \& Mollo, 2007). Essas alterações nas concentrações circulantes dos hormônios esteroides observadas em vacas de alta produção leiteira, provavelmente são responsáveis por um maior tempo necessário para ovulação após luteólise neste grupo de animais em relação a novilhas ou vacas secas (Sartori et al., 2002a). As razões para menores concentrações de hormônios circulantes em vacas com maior IMS estão, provavelmente, relacionadas à menor produção pelos ovários ou a maior metabolismo destes hormônios, sendo a primeira hipótese pouco provável e não apropriadamente testada até o momento. Por outro lado, Sangsritavong et al. (2002) demonstraram que vacas lactantes (com maior ingestão alimentar) têm um metabolismo de hormônios esteroides muito maior do que não lactantes (com menor ingestão). Foi demonstrado, inclusive, que alta IMS causa um aumento do fluxo sanguíneo hepático (Parr et al., 1993; Sangsritavong et al., 2002) onde grande quantidade dos hormônios esteroides é metabolizada. Consequentemente, com o aumento da IMS há redução nas concentrações circulantes desses hormônios. Tais observações foram descritas, por exemplo, por Nolan et al. (1998) e Santos (2005) que constataram maiores concentrações circulantes de progesterona em novilhas ou vacas secas dos grupos experimentais sob menor IMS/energia.

Dois estudos no Brasil com fêmeas da raça Nelore sob baixa $(0,7 \mathrm{M})$ ou alta $(1,7 \mathrm{M})$ IMS avaliaram características do ciclo estral tanto em novilhas (Mollo et al., 2007) quanto em vacas não lactantes (Martins et al., 2008). No primeiro trabalho, as novilhas submetidas à alta IMS ovularam folículos maiores e formaram CLs maiores sem, entretanto, apresentarem diferenças nas concentrações circulantes de estradiol e progesterona. No segundo estudo, apesar de não ter sido detectada diferença no tamanho do folículo ovulatório entre vacas com alta e baixa IMS, as concentrações séricas pré-ovulatórias de estradiol foram maiores nas vacas com baixa IMS. Em ambos os estudos as concentrações circulantes de insulina foram mais altas nas fêmeas sob alta IMS.

Alterações na ingestão de energia também influenciam a reprodução através de mudanças na duração e intensidade de estro em bovinos. Em estudos com bovinos leiteiros que compararam animais com menor IMS/energia devido à ausência de lactação (novilhas nulíparas) versus fêmeas com alta IMS (vacas lactantes; Nebel et al., 1997) ou vacas com menor ( $<39,5 \mathrm{~kg} / \mathrm{dia})$ versus maior $(>39,5 \mathrm{~kg} / \mathrm{dia})$ produção leiteira (Lopez et al., 2004), houve sempre maior número de montas e duração de estro mais prolongada nas fêmeas com menor IMS (novilhas ou vacas com menor produção leiteira). Esse efeito da IMS nas características do estro não é exclusivo de fêmeas de raças leiteiras. Em um trabalho com novilhas Nelore nulíparas, onde a única diferença entre os grupos experimentais foi a quantidade de dieta fornecida, os animais submetidos à restrição alimentar $(0,7 \mathrm{M})$ apresentaram duração de estro mais prolongada (17 vs 11 horas) e comportamento de cio mais intenso, caracterizado por três vezes mais aceites de monta quando comparadas às novilhas superalimentadas (1,7M; Mollo et al., 2007). 
Como mencionado anteriormente, além do efeito sobre as características do ciclo estral em bovinos, a alta IMS também parece afetar a eficiência reprodutiva das fêmeas. O fornecimento de alta quantidade de alimento resultou em redução no desenvolvimento embrionário tanto in vivo quanto in vitro na maioria dos estudos com fêmeas ruminantes superovuladas ou submetidas à aspiração folicular para produção in vitro de embriões (revisado por Sartori et al., 2007). Em vacas com ovulação natural, alta ingestão alimentar associada à produção de leite elevada também foi associada à menor viabilidade embrionária (Sartori et al., 2002b).

Dentre os inúmeros fatores envolvidos com a queda de fertilidade ou redução na qualidade embrionária em bovinos submetidos à alta ingestão alimentar, há dois que se destacam. O primeiro é que animais com elevado ECC ou consumindo dietas com altos teores energéticos podem apresentar um quadro de hiperinsulinemia ou resistência à insulina que está relacionado à queda na qualidade ovocitária (Adamiak et al., 2005). Além disso, essas fêmeas, geralmente, têm concentrações circulantes elevadas de glicose e IGF-I, o que pode interferir no transporte de glicose nos embriões e aumentar a apoptose (Santos et al., 2008). O segundo fator é em decorrência da redução nas concentrações circulantes de hormônios esteroides, principalmente estradiol e progesterona nos animais com alta IMS/energia.

Alterações nas concentrações sanguíneas de progesterona e estradiol podem afetar padrões de crescimento folicular (Sirois \& Fortune, 1988; Knopf et al., 1989), qualidade ovocitária e viabilidade embrionária (Folman et al., 1973; Fonseca et al., 1983; Mann et al., 1998). Algumas das possíveis explicações para a redução da qualidade ovocitária/embrionária em animais com reduzidas concentrações circulantes de estradiol e progesterona estão relacionadas ao período mais prolongado de crescimento do folículo pré-ovulatório após a luteólise, ou seja, um período mais longo de dominância folicular previamente à ovulação. Isso ocorre, provavelmente pela necessidade de o folículo préovulatório crescer por um período mais longo e produzir estradiol suficiente para compensar o que é metabolizado em excesso e induzir o pico ovulatório de GnRH. Um trabalho recente (Cerri et al., 2009a) com vacas holandesas lactantes demonstrou maior proporção de embriões degenerados naqueles provenientes de ovulações onde a dominância folicular foi mais prolongada. Em contrapartida, folículos que ovularam mais precocemente após o estabelecimento da dominância, produziram maior porcentagem de embriões viáveis.
O metabolismo aumentado do hormônio progesterona também pode estar relacionado com a queda na fertilidade em fêmeas superalimentadas. Reduzidas concentrações séricas de progesterona antes da IA foram associadas à queda de fertilidade em vacas (Folman et al., 1973; Fonseca et al., 1983). Da mesma forma, vacas doadoras de ovócitos com concentrações circulantes muito baixas de progesterona tiveram menor taxa de clivagem e menor produção in vitro de embriões do que aquelas com concentrações sanguíneas mais elevadas de progesterona (Pfeifer et al., 2009). Baixas concentrações séricas de progesterona levam a um aumento na freqüência de pulsos de LH (Roberson et al., 1989; Adams et al., 1992), podendo causar maturação prematura dos ovócitos (Revah \& Butler, 1996), queda na qualidade ovocitária no momento da ovulação e consequente qualidade embrionária inferior após a fecundação (Ahmad et al., 1995). Concentrações reduzidas de progesterona após a IA também estão associadas à fertilidade reduzida (Mann et al., 1995; Larson et al., 1997). Essa redução na fertilidade pela baixa progesterona pode ser devido a um desenvolvimento embrionário retardado (Mann et al., 1998; Mann \& Lamming, 2001; Lonergan et al., 2007; Carter et al., 2008). Estudos em vacas e ovelhas mostraram que suplementação de progesterona durante os primeiros 4 a 6 dias após a IA aumentou o crescimento embrionário ou fetal (Garrett et al., 1988; Kleemann et al., 1994). Outros estudos em bovinos obtiveram resultados semelhantes, pois com a elevação das concentrações circulantes de progesterona através do uso de dispositivos intravaginais de progesterona no dia 3 do ciclo estral (Carter et al., 2008) ou através da superovulação da doadora/receptora (Lonergan et al., 2007), os autores obtiveram aumento no crescimento embrionário.

Influência da gordura na reprodução de fêmeas bovinas

A suplementação de gordura é uma prática comum para aumentar a densidade energética da dieta, principalmente em gado de leite. Entretanto, além de fornecer calorias, a gordura possui efeito direto na reprodução pela ação dos ácidos graxos presentes em sua composição (Funston, 2004; Raes et al., 2004).

As principais famílias de ácidos graxos polinsaturados (AGPs) que afetam a fertilidade são n-3 e n-6. O ácido linoleico dietético é um dos representantes da família n-6 (Wathes et al., 2007), o qual é convertido em ácido aracdônico, precursor das prostaglandinas dienoicas, como a PGF2 $\alpha$. Por outro lado, o ácido linolênico (n-3) é convertido a ácido eicosapentaenoico precursor das prostaglandinas trienoicas, como PGF3 $\alpha$. Deste modo, a produção das 
prostaglandinas pode ser manipulada de acordo com a proporção de ácidos graxos presentes na dieta.

Os AGPs podem elevar a fertilidade em bovinos por diferentes caminhos. Nos ovários, há relatos de efeito dos ácidos graxos sobre o número e tamanho de folículos ovarianos (Lucy et al., 1991; Robinson et al., 2002; Bilby et al., 2006), tamanho do CL (Raes et al., 2004; Bilby et al., 2006) e na qualidade ovocitária (Kim et al., 2001; Zeron et al., 2002). Além disso, outros estudos também observaram influência de dietas contendo ácidos graxos sobre a qualidade embrionária (Kojima et al., 1997; Childs et al., 2008a; Cerri et al., 2009b) e a concentração circulante de prostaglandinas e hormônios esteroides (Ryan et al., 1992; Thomas et al., 1997; Petit et al., 2002; Childs et al., 2008b).

A causa da associação observada entre suplementação com lipídeos e maiores concentrações circulantes de hormônios esteroides relatada por diversos autores não está bem definida. Especula-se que pode ser por meio da ação dos AGPs, aumentando a pulsatilidade de LH (Hightshoe et al., 1991), com um consequente aumento no tamanho do folículo pré-ovulatório e subsequente CL (Raes et al., 2004), ou pela diminuição do metabolismo hepático desses hormônios (Hawkins et al., 1995). Outra possibilidade seria aumentando as concentrações circulantes de colesterol, precursor da síntese de esteroides (Thomas et al., 1997) ou mesmo alterando a síntese de prostaglandina (Petit \& Twagiramungu, 2006; Childs et al., 2008b).

A teoria da diminuição do metabolismo dos esteroides é atualmente uma das mais discutidas para explicar as maiores concentrações de esteroides na suplementação lipídica. Hawkins et al. (1995) avaliaram o mecanismo pelo qual a inclusão de gordura na dieta aumenta a concentração de progesterona circulante, através do fornecimento de sais de cálcio de ácidos graxos de cadeia longa, iniciando 100 dias antes do parto. A concentração de progesterona foi maior para novilhas de corte mantidas nesta dieta, porém as concentrações luteais de progesterona foram similares entre os grupos, sugerindo que não houve diferença na taxa de secreção de progesterona. Por outro lado, o tempo requerido para a queda pela metade das concentrações circulantes de progesterona, após ovariectomia, foi maior nas novilhas suplementadas com gordura. Os autores sugeriram, então, que em vacas alimentadas com dieta rica em lipídios, o fator principal relacionado ao aumento das concentrações circulantes de progesterona seria a redução no metabolismo hepático. Adicionalmente, Piccinato et al. (2010) observaram in vitro que ácidos graxos com cadeias de carbono de diferentes comprimentos diminuíram o metabolismo de esteroides. Entretanto, esses resultados não foram corroborados por um experimento subsequente realizado in vivo, através de infusão de óleo de linhaça no abomaso. Por outro lado, ainda não foi realizado um estudo de maneira precisa para calcular o fluxo sanguíneo hepático nestas condições experimentais.

Em experimento realizado por nossa equipe o metabolismo hepático de progesterona de 38 novilhas Nelore pré-púberes foi avaliado indiretamente, após 15 dias de suplementação com concentrados isoenergéticos e isoproteicos contendo gordura protegida ruminal (100 g/d de Megalac- $\mathrm{E}^{\circledR}$; 18 animais) e sem gordura (20 animais) em um delineamento "cross-over". Neste estudo, as concentrações séricas de progesterona 4 dias após a inserção de um dispositivo intravaginal de progesterona (CIDR) foi maior para o grupo que recebeu dieta com gordura do que o grupo controle (3,8 \pm 0,3 e 2,8 \pm 0,3, respectivamente; $\mathrm{P}=0,008)$. Concordando com esta hipótese, a suplementação com Megalac-E provavelmente reduziu o metabolismo hepático de progesterona em vacas mestiças não lactantes e ovariectomizadas, por apresentarem maiores concentrações plasmáticas de progesterona em relação àquelas que não receberam esta gordura na dieta (1,81 vs 1,65 ng/mL; P = 0,01; Lopes et al., 2009).

Com relação às estruturas ovarianas, vacas de leite suplementadas com dietas ricas em AGPs ou que receberam infusão de gordura no abomaso tiveram folículos dominantes maiores (Staples et al., 1998; Robinson et al., 2002). Da mesma forma, Staples et al. (2000) detectaram um maior tamanho do folículo ovulatório em vacas de leite suplementadas com AGPs, em comparação às que receberam ácido oleico na dieta. Em outros estudos, maiores folículos pré-ovulatórios também foram relatados em vacas de leite que receberam ácido linolênico (Ambrose et al., 2006; Bilby et al., 2006) e linoleico (Bilby et al., 2006) em relação a grupos controle. Por outro lado, um estudo do nosso laboratório não detectou diferença no tamanho do folículo ovulatório e no CL 7 e 14 dias após ovulação de novilhas Nelore suplementadas ou não com Megalac-E. Concordando com estes resultados, dietas ricas em ácido linoleico ou linolênico não alteraram o tamanho do folículo dominante (Ambrose et al., 2006), o diâmetro e a taxa de crescimento do folículo pré-ovulatório (Childs et al., 2008b), ou o tamanho do CL e a concentração plasmática de progesterona (Ambrose et al., 2006; Childs et al., 2008b). Vacas leiteiras alimentadas com sais de cálcio de ácido graxo linoleico também apresentaram diâmetros similares do folículo pré-ovulatório e luteal, em comparação aos animais que receberam sais de cálcio de óleo de palma (Cerri et al., 2009b). Da mesma forma, os suplementos lipídicos enriquecidos com ácidos graxos 
n-9 cis (18:1c, oleico), n-9 trans (18:1t), n-6 (18:2, linoleico, Megalac-R) ou n-3 (18:3, linolênico) não interferiram nas concentrações circulantes de progesterona (Bilby et al., 2006).

Relativamente, poucos trabalhos avaliaram o efeito dos ácidos graxos na produção e desenvolvimento de embriões produzidos in vivo (Ryan et al., 1992; Kojima et al., 1997; Bader et al., 2005; Thangavelu et al., 2007; Childs et al., 2008a; Petit et al., 2008; Cerri et al., 2009b). Além disso, ainda não há consenso se os ácidos graxos são mais importantes para o embrião em si ou para sua implantação (Petit et al., 2008), pois, podem reduzir a síntese ovariana e endometrial de PGF2 $\alpha$, auxiliar o embrião a produzir interferon t, bem como alterar o ambiente do oviduto e do útero. Esses efeitos contribuem para o desenvolvimento embrionário, redução na morte embrionária e consequente estabelecimento da prenhez (Binelli et al., 2001; Mattos et al., 2004; Childs et al., 2008a). Nesse sentido, foi demonstrado que a inclusão de AGPs na suplementação dietética teve efeito positivo no desenvolvimento embrionário (Thangavelu et al., 2007). Vacas de leite alimentadas com sais de cálcio de ácido graxo linoleico apresentaram maiores porcentagens de embriões de alta qualidade $(73,5 \%$ vs $51,5 \%, P=0,06)$ em relação aos animais que receberam sais de cálcio de óleo de palma (Cerri et al., 2009b). Da mesma forma, em novilhas de corte, o número de embriões degenerados foi reduzido para os animais que receberam dieta rica em AGPs n-3 em relação à dieta com ácido graxo saturado (Childs et al., 2008a).

Por outro lado, um estudo recente (Guardieiro et al., 2010) não observou efeito positivo da suplementação com Megalac-E na produção de embriões em doadoras da raça Nelore. Este resultado associado às taxas de prenhez encontradas em animais suplementados com ácido linoleico após IA e inovulação de embriões reforçam a hipótese da sua atuação no útero, auxiliando em mecanismos antiluteolíticos. A infusão no abomaso de gordura contendo alta quantidade de ácido linoleico (Oldick et al., 1997) ou a suplementação com AGPs n-6 protegidos (Cheng et al., 2001) provocaram menor liberação de PGF2 $\alpha$ em resposta à ocitocina. Lopes et al. (2009) forneceram Megalac-E durante o início do protocolo de sincronização do estro até 28 dias após IATF e observaram em vacas Nelore lactantes, taxas de prenhez superiores $(51,2 \%, n=451)$, em relação ao grupo não suplementado (39,6\%, $\mathrm{n}=459 ; \mathrm{P}=0,04)$. Este mesmo grupo realizou um experimento com suplementação entre o momento da IA até o diagnóstico de gestação e também obtiveram maior taxa de concepção para vacas que receberam Megalac-E (48,2\%, 81/168) em relação às que receberam Megalac $(33,9 \%, 58 / 177)$ ou àquelas suplementadas com mineral proteico (26,4\%, 63/238; Lopes et al., 2008). Dieta com semente de girassol (rica em linoleico) foi fornecida a vacas Nelore pós-parto $(n=133)$ do momento da IA até 22 dias após o protocolo e observou-se aumento de $20,4 \%$ na taxa de concepção para vacas que receberam esta dieta (Peres et al., 2008).

Adicionando Megalac-E à suplementação mineral proteica de receptoras de embrião mestiças $(n=435)$ do final do protocolo de sincronização até 21 dias após a inovulação, houve um acréscimo na taxa de concepção de 11,9\% (49,6\% vs 37,7\%) em relação ao grupo controle (Lopes et al., 2009). Desta forma, esses autores verificaram que os AGPs possuem efeito pós inseminação, especificamente durante o período de luteólise.

Apesar de os resultados acima de suplementação com gordura protegida terem sido favoráveis à fertilidade de vacas de corte, outros estudos da nossa equipe e de outros pesquisadores não observaram esse mesmo efeito positivo nessa categoria animal, principalmente quando a suplementação foi iniciada diversos dias antes da IA ou transferência de embriões (dados não publicados).

\section{Proteína e reprodução na vaca leiteira}

Devido ao fato de haver correlação positiva entre níveis de proteína bruta (PB) na dieta e produção de leite, dietas com alto teor de $\mathrm{PB}$ (>18\%) são comumente utilizadas no pós-parto de vacas de alta produção leiteira. Alta concentração de PB na dieta, entretanto, tem sido associada à redução no desempenho reprodutivo (Butler, 1998; Santos \& Amstalden, 1998; Westwood et al., 1998; Ferreira et al., 2008). Dieta com excesso de PB ou a suplementação direta de ureia como fonte de nitrogênio não proteico (NNP) pode resultar no aumento da concentração plasmática de ureia (Canfield et al., 1990). A ureia é uma molécula relativamente pequena que tem a habilidade de atravessar as membranas celulares facilmente, incluindo no trato reprodutivo (O’Callaghan \& Boland, 1999).

O estabelecimento de uma gestação depende de uma série de eventos inter-relacionados, tais como, desenvolvimento folicular e ovulação, fecundação, transporte e desenvolvimento do embrião, reconhecimento materno da gestação e placentação. Potencialmente, amônia, ureia ou algum outro produto tóxico do metabolismo da proteína podem interceder em um ou mais desses passos e prejudicar a eficiência reprodutiva (Butler, 1998). A amônia é um metabólito da proteína que escapa da detoxificação pelo sistema hepático do ciclo da ureia. Já a ureia, é outro metabólito da proteína da dieta, que é formada da detoxificação da amônia pelo fígado. A concentração de ureia no plasma ou soro é refletida pela quantidade e 
degradabilidade ruminal da proteína consumida, pela severidade do BEN, ou pela combinação de consumo de proteína e BEN (Elrod \& Butler, 1993). Além disso, elevadas concentrações plasmáticas de ureia poder ocorrer em decorrência do fornecimento de dietas com teores altos de NNP ou PB associados a quantidades insuficientes de carboidratos disponíveis para fermentação no rúmen. Portanto, os efeitos combinados do excesso de proteína degradável no rúmen (PDR), estado energético ou fornecimento inadequado de carboidratos poderiam, em parte, justificar a baixa qualidade embrionária observada principalmente em vacas em lactação (Sartori et al., 2002b). Nesse sentido, Butler (1998) observou que a taxa de desenvolvimento embrionário foi reduzida em vacas lactantes alimentadas com excesso de PDR. Em outro estudo, entretanto, não houve diferença na produção de embriões viáveis em vacas lactantes superovuladas que possuíam moderada $(15,5 \mathrm{mg} / \mathrm{dL})$ ou alta $(24,4 \mathrm{mg} / \mathrm{dL})$ concentração plasmática de nitrogênio ureico (Rhoads et al., 2006). Quando esses embriões foram transferidos para receptoras, contudo, houve maior taxa de concepção naquelas que receberam os embriões das vacas com moderada concentração plasmática de nitrogênio ureico (35 vs $11 \%$ ). Além disso, os autores não detectaram diferença na taxa de concepção entre receptoras com moderada ou alta concentração plasmática de nitrogênio ureico. Outro estudo em vacas superovuladas (Dawuda et al., 2002) observou efeito negativo na produção de embriões apenas nas vacas que receberam suplementação com ureia entre a IA e a colheita dos embriões. Vacas controle ou aquelas suplementadas com ureia por um período mais prolongado (a partir de 10 dias antes da IA) não tiveram comprometimento na produção ou qualidade dos embriões. Em experimentos in vitro com concentrações elevadas de ureia e amônia, também observou-se diminuição no desenvolvimento embrionário (Ocon \& Hansen, 2003). Além disso, estudos mostraram menor taxa de clivagem e menos embriões viáveis obtidos de doadoras de ovócitos que apresentaram maiores concentrações plasmáticas de ureia e amônia (Sinclair et al., 2000; Armstrong et al., 2001). Menores taxas de concepção ao primeiro serviço também foram observadas em vacas lactantes e novilhas com concentração sérica de ureia acima de $20 \mathrm{mg} / \mathrm{dL}$ (descréscimo de 30 e 20\%, respectivamente; Leroy et al., 2008b). No entanto, não está claro se o aumento das concentrações de ureia circulantes, através da manipulação da dieta, é responsável por provocar os mesmos efeitos deletérios em ovócitos e embriões produzidos in vivo (Santos et al., 2008).
A baixa sobrevivência embrionária em vacas lactantes pode também ser devido à menor concentração circulante de progesterona. De fato, vacas lactantes recebendo dietas com elevada PB tiveram menores concentrações circulantes de progesterona quando comparadas àquelas alimentadas com níveis inferiores de PB. Nesse mesmo levantamento, entretanto, essa diferença não foi observada em vacas não lactantes (Blauwiekel et al., 1986) ou em outro estudo com novilhas (Elrod \& Butler, 1993). Essas concentrações circulantes mais baixas de progesterona em vacas de elevada produção leiteira alimentadas com alto teor de PB, entretanto, podem ser devido à maior IMS nesses animais, o que acarreta maior metabolismo hepático dos hormônios esteroides (Sangsritavong et al., 2002), e não necessariamente aos teores de PB por si só.

O excesso de proteína na dieta pode afetar a reprodução por produzir efeitos diretos no ambiente uterino, pois os subprodutos do seu metabolismo (mais especificamente os íons de amônia) provocam a redução do pH luminal uterino durante a fase lútea inicial, alterando a secreção das glândulas endometriais, o que pode estar relacionada com a redução nas taxas de concepção (Elrod et al., 1993; Butller, 1998). Redução da fertilidade e perda embrionária podem ocorrer quando uma dieta com ureia é oferecida em excesso (McEvoy et al., 1997) ou quando é aumentada a PDR (Blanchard et al., 1990). Assim, Butller (1998) especula que os efeitos negativos da alta proteína podem ser mediados pelo decréscimo no $\mathrm{pH}$ uterino que, em combinação com baixa progesterona circulante, criam um ambiente uterino hostil ao embrião.

Embora diversos estudos tenham demonstrado uma relação entre alimentação com altos teores de PB e queda na fertilidade em vacas de alta produção, pouco se sabe realmente dos mecanismos envolvidos neste processo. De qualquer forma, em termos gerais, acredita-se que dietas que não produzam concentrações plasmáticas de nitrogênio ureico acima de $20 \mathrm{mg} / \mathrm{dL}$ não parecem comprometer a fertilidade de vacas leiteiras (Ferguson, 1991; Ferguson et al., 1993).

\section{Considerações Finais}

A nutrição se torna fundamental no desempenho reprodutivo, uma vez que pode afetar direta ou indiretamente aspectos da fisiologia da fêmea bovina por meio principalmente da energia, proteína e gordura presentes na dieta. Além disso, o desempenho reprodutivo também está associado ao ECC no período pós-parto, o qual pode ser 
alterado pela suplementação alimentar no intuito de acelerar o reinício da ciclicidade nas vacas pós-parto, via restabelecimento da pulsatilidade normal de $\mathrm{LH}$.

Ao se considerar os efeitos da alta IMS/energia sobre a fertilidade bovina, destacam-se os negativos, pois estão associados a um metabolismo elevado dos hormônios esteroides e aumento nas concentrações circulantes de insulina e IGF-I, refletindo em um possível comprometimento na qualidade ovocitária/embrionária e menores taxas de concepção.

A utilização de altos teores de proteína ou NNP na dieta também apresenta aspectos negativos à reprodução, pois elevam as concentrações plasmáticas de ureia e amônia, diminuindo o $\mathrm{pH}$ luminal uterino durante a fase lútea inicial e alteram a secreção das glândulas endometriais. Por outro lado, a suplementação lipídica com AGPs potencialmente melhora a função reprodutiva em fêmeas bovinas, provavelmente por inibir a resposta luteolítica e aumentar as concentrações circulantes de progesterona, contribuindo para a manutenção da prenhez.

Em razão de fatores nutricionais como energia (IMS e gordura) e proteína presentes na dieta influenciarem positiva ou negativamente a reprodução bovina, estratégias de suplementação dietética podem ser adotadas de maneira efetiva para obter resultados favoráveis à fertilidade da fêmea bovina.

\section{Referências}

ADAMIAK, S.J.; MACKIE, K.; WATT, R.G. et al. Impact of nutrition on oocyte quality: cumulative effects of body composition and diet leading to hyperinsulinemia in cattle. Biology of Reproduction, v.73, p.918-926, 2005.

ADAMS, G.P.; MATTERI, R.L.; KASTELIC, J.P. et al. Association between surges of follicle-stimulating hormone and the emergency of follicular waves in heifers. Journal of Reproduction and Fertility, v.94, p.177-188,1992.

AHMAD, N.; SCHRICK, F.N.; BUTCHER, R.L. et al. Effect of persistent follicles on early embryonic losses in beef cows. Biology of Reproduction, v.52, p.1129-1135, 1995.

AMBROSE, D.J.; KASTELIC, J.P.; CORBETT, R. et al. Lower pregnancy losses in lactating dairy cows fed a diet enriched in á-linolenic acid. Journal of Dairy Science, v.89, p.30663074, 2006.

ARÉCHIGA, C.F.; ORTÍZ, O.; HANSEN, P.J. Effect of prepartum injection of vitamin $E$ and selenium on postpartum reproductive function of dairy cattle. Theriogenology, v.41, p.1251-1258, 1994.

ARÉCHIGA, C.F.; VAZQUEZ-FLORES, S.; ORTIZ, O. et al. Effect of injection of beta-carotene or vitamin $E$ and selenium on fertility of lactating dairy cows. Theriogenology, v.50, p.65-76, 1998.

ARMSTRONG, D.G.; MC EVOY, T.G.; BAXTER, G. et al. Effect of dietary energy and protein on bovine follicular dynamics and embryo production in vitro: associations with the ovarian insuline-like growth factor system. Biology of Reproduction, v.64, p.1624-1632, 2001.
BADER, J.F.; KOJIMA, F.N.; WEHRMAN, M.E. et al. Effects of prepartum lipid supplementation on FSH superstimulation and transferable embryo recovery in multiparous beef cows. Animal Reproduction Science, v.85, p.61-70, 2005.

BALL, P.J.H.; MCEWAN, E.E.A. The incidence of prolonged luteal function following early resumption of ovarian activity in post partum dairy cows. In: BRITISH SOCIETY OF ANIMAL SCIENCE. Proceedings... Edimburgh: BSAS, 1998. p.187. Resumo.

BARUSELLI, P.S.; REIS, E.L.; MARQUES, M.O. et al. The use of hormonal treatments to improve reproductive performance of anestrous beef cattle in tropical climates. Animal Reproduction Science, v.82-83, p.479-486, 2004.

BEAM, S.W.; BUTLER, W.R. Energy balance, metabolic hormones, and early postpartum follicular development in dairy cows fed prilled lipid. Journal of Dairy Science, v.81, p.121-131, 1998.

BILBY, T.R.; BLOCK, J.; DO AMARAL, B.C. et al. Effects of dietary unsaturated fatty acids on oocyte quality and follicular development in lactating dairy cows in summer. Journal of Dairy Science, v.89, p.3891-3903, 2006.

BINELLI, M.; THATCHER, W.W.; MATTOS, R. et al. Antiluteolytic strategies to improve fertility in cattle. Theriogenology, v.56, p.1451-1463, 2001.

BLANCHARD, T.; FERGUSON, J.; LOVE, L. et al. Effect of dietary crude-protein type on fertilization and embryo quality in dairy cattle. American Journal of Veterinary Research, v.51, p.905-908, 1990.

BLAUWIEKEL, R.; KINCAID, R.L.; REEVES, J.J. Effect of high crude protein on pituitary and ovarian function in Holstein cows. Journal of Dairy Science, v.69, p.439-446, 1986.

BOSSIS, I.; WETTEMANN, R.P.; WELTY, S.D. et al. Nutritionally induced anovulation in beef heifers: ovarian and endocrine function during realimentation and resumption of ovulation. Biology of Reproduction, v.62, p.1436-1444, 2000.

BUTLER, W.R. Review: effect of protein nutrition on ovarian and uterine physiology in dairy cattle. Journal of Dairy Science, v.81, p.2533-2539, 1998.

BUTLER, W.R.; SMITH, R.D. Interrelationships between energy balance on postpartum reproductive function in dairy cattle. Journal of Dairy Science, v.7, p.767-783, 1989.

CANFIELD, R.W.; SNIFFEN, C.J.; BUTLER, W.R. Effects of excess degradable protein on postpartum and energy balance in dairy cattle. Journal of Dairy Science, v.73, p.2342-2340, 1990.

CARTER, F.; FORDE, N.; DUFFY, P. et al. Effect of increasing progesterone concentration from Day 3 of pregnancy on subsequent embryo survival and development in beef heifers. Reproduction, Fertility and Development, v.20, p.368375, 2008.

CERRI, R.L.; RUTIGLIANO, H.M.; CHEBEL, R.C. et al. Period of dominance of the ovulatory follicle influences embryo quality in lactating dairy cows. Reproduction, v.137, p.813-823, 2009a.

CERRI, R.L.A.; JUCHEM, S.O.; CHEBEL, R.C. et al. Effect of fat source differing in fatty acid profile on metabolic parameters, fertilization, and embryo quality in high-producing dairy cows. Journal of Dairy Science, v.92, p.1520-1531, 2009 b.

CHENG, Z.; ROBINSON, R.S.; PUSHPAKUMARA, P.G.A. et al. Effect of dietary polyunsaturated fatty acids on uterine prostaglandin synthesis in the cow. Journal of Endocrinology, v.171, p.463-473, 2001.

CHILDS, S.; CARTER, F.; LYNCH, C.O. et al. Embryo yield and quality following dietary supplementation of beef heifers with n-3 polyunsaturated fatty acids (PUFA). Theriogenology, v.70, p.992-1003, 2008a.

CHILDS, S.; LYNCH, C.O.; HENNESSY, A.A. et al. Effect of dietary enrichment with either n-3 or n-6 fatty acids on systemic metabolite and hormone concentration and ovarian function in heifers. Animal, v.2, p.883-893, 2008b. 
DARWASH, A.O.; LAMMING, G.E.; WOOLLIAMS, J.A. The phenotypic association between the interval to post-partum ovulation and traditional measures of fertility in dairy cattle. Animal Science, v.65, p.9-16, 1997.

DAWUDA, P.M.; SCARAMUZZI, R.J.; LEESE, H.J. et al. Effect of timing of urea feeding on the yield and quality of embryos in lactating dairy cows. Theriogenology, v.58, p.1443-1455, 2002.

DISKIN, M.G.; MORRIS, D.G. Embryonic and early foetal losses in cattle and other ruminants. Reproduction in Domestic Animals, v.43(Suppl. 2), p.260-267, 2008.

ELROD, C.C.; BUTLER, W.R. Reduction of fertility and alteration of uterine $\mathrm{pH}$ in heifers fed excess ruminally degradable protein. Journal of Animal Science, v.71, p.694-701, 1993.

FERGUSON, J.D. Nutrition and reproduction in dairy cows. Veterinary Clinics of North America Food Animal Practice, v.7, p.483-507, 1991

FERGUSON, J.D.; GALLIGAN, D.T.; BLANCHARD, T. et al. Serum urea nitrogen and conception rate: the usefulness of test information. Journal of Dairy Science, v.76, p.3742-3746, 1993.

FERREIRA, F.A.; BINELLI, M.; RODRIGUES, P.H.M. Interação entre nutrição protéica e aspectos reprodutivos em fêmeas bovinas. Revista Brasileira de Reprodução Animal, v.32, p.67-79, 2008.

FOLMAN, Y.; ROSENBER, M.; HERZ, Z. et al. Relationship between plasma progesterone concentration and conception in postpartum dairy-cows maintained on two levels of nutrition. Journal of Reproduction and Fertility, v.34, p.267-278, 1973.

FONSECA, F.A.; BRITT, J.H.; MCDANIEL, B.T. et al. Reproductive traits of holsteins and jerseys - effects of age, milkyield, and clinical abnormalities on involution of cervix and uterus, ovulation, estrous cycles, detection on estrus, conception rate, and days open. Journal of Dairy Science, v.66, p.1128-1147, 1983.

FUNSTON, R.N. Fat supplementation and reproduction in beef females. Journal of Animal Science, v.82(Suppl.), p.154$161,2004$.

GARRETT, J.E.; GEISERT, R.D.; ZAVY, M.T. et al. Evidence for maternal regulation of early conceptus growth and development in beef cattle. Journal of Reproduction and Fertility, v.84, p.437-446, 1988.

GRIMARD, B.; HUMBLOT, P.; PONTER, A.A. et al. Influence of postpartum energy restriction on energy status, plasma LH and oestradiol secretion and follicular development in suckled beef cows. Journal of Reproduction and Fertility, v.104, p.173179, 1995.

GUARDIEIRO, M.M.; MACHADO, G.M.; BASTOS, M.R. et al. Post cryopreservation viability of embryos from Nellore heifers supplemented with rumen-protected fat. Reproduction, Fertility and Development, v.22, p.205-206, 2010. (abstr.).

HAWKINS, D.E.; NISWENDER, K.D.; OSS, G.M. et al. An increase in serum lipids increases luteal lipid content and alters the disappearance rate of progesterone in cows. Journal of Animal Science, v.73, p. 541-545, 1995.

HIGHTSHOE, R.B.; COCHRAN, R.C.; CORAH, L.R. et al. Effects of calcium soaps of fatty acids on postpartum reproductive function in beef cows. Journal of Animal Science, v.69, p.4097-4103, 1991.

INGRAHAM, R.H.; KAPPEL, L.C.; MORGAN, E.B. et al. Correction of subnormal fertility with copper and magnesium supplementation. Journal of Dairy Science, v.70, p.167$180,1987$.

KIM, M.; KINOSHITA, J.Y. Lipid and fatty acid analysis of fresh and frozen-thawed immature and in vitro matured bovine oocytes. Reproduction, v.122, p.131-138, 2001.

KLEEMANN, D.O.; WALKER, S.K.; SEAMARK, R.F. Enhanced fetal growth in sheep administered progesterone during the first three days of pregnancy. Journal of Reproduction and Fertility, v.102, p.411-417, 1994.

KNOPF, L.; KASTELIC, J.P.; SCHALLENBERGER, E. et al. Ovarian follicular dynamics in heifers: test of two wave hypothesis by ultrasonically monitoring individual follicles. Domestic Animal Endocrinology, v.6, p.111-119, 1989.

KOJIMA, T.; ZENIYA, Y.; AOYAMA, T. et al. Dietary administration of fatty acids-enriched mold dried cell containing ã-linolenic acid to female pigs improves ovulation rate and embryo quality in summer. Journal of Reproduction and Development, v.43, n.2, p.121-127, 1997.

LAMMING, G.E.; DARWASH, A.O. The use of milk progesterone profiles to characterize components of subfertility in milked dairy cows. Animal Reproduction Science, v.52, p.175-190, 1998.

LARSON, S.F.; BUTLER, W.R.; CURRIE, W.B. Reduced fertility associated with low progesterone postbreeding and increased milk urea nitrogen in lactating cows. Journal of Dairy Science, v.80, p.1288-1295, 1997.

LEROY, J.L.; VANHOLDER, T.; MATEUSEN, B. et al. Nonesterified fatty acids in follicular fluid of dairy cows and their effect on developmental capacity of bovine oocytes in vitro. Reproduction, v.130, p.485-495, 2005.

LEROY, J.L.; OPSOMER, G.; VAN SOOM, A. et al. Reduced fertility in high-yielding dairy cows: are the oocyte and embryo in danger? Part I.The importance of negative energy balance and altered corpus luteum function to the reduction of oocyte and embryo quality in high-yielding dairy cows. Reproduction in Domestic Animals, v.43, p.612-622, 2008a.

LEROY, J.L.M.R.; VAN SOOM, A.; OPSOMER, G. et al. Reduced fertility in high-yielding dairy cows: Are the oocyte and embryo in danger? Part II. Mechanisms linking nutrition and reduced oocyte and embryo quality in high-yielding dairy cows. Reproduction in Domestic Animals, v.43, p.623-632, 2008b.

LONERGAN, P.; WOODS, A.; FAIR, T. et al. Effect of embryo source and recipient progesterone environment on embryo development in cattle. Reproduction, Fertility and Development, v.19, p.861-868, 2007.

LOPES, C.N.; SCARPA, A.B.; CAPPELLOZZA, B.I. et al. Effects of rumen-protected polyunsaturated fatty acid supplementation on reproductive performance of Bos indicus beef cows. Journal of Animal Science, v.87, p.3935-3943, 2009. doi:10.2527/ jas.2009-2201.

LOPES, C.N.; ZILIOTI, C.; ARAÚJO, T. et al. Efeito do tratamento do Megalac e Megalac-E na taxa de prenhez em vacas Nelore pós-parto submetidas a IATF. Acta Scientiae Veterinariae, v.36, suppl.2, p.616, 2008. (abstr.).

LÓPEZ-GATIUS, F.; SANTOLARIA, P.; YANIZ, J. et al. Factors affecting pregnancy loss from gestation Day 38 to 90 in lactating dairy cows from a single herd. Theriogenology, v.57, p.1251-1261, 2002.

LOPEZ, H.; SATTER, L.D.; WILTBANK, M.C. Relationship between level of milk production and estrous behavior of lactating dairy cows. Animal Reproduction Science, v.81, p.209-223, 2004.

LUCY, M.C.; STAPLES, C.R.; MICHEL, F.M. et al. Effect of feeding calcium soaps to early postpartum dairy cows on plasma prostaglandin $\mathrm{F}_{2 a ́}$, luteinizing hormone, and follicular growth. Journal of Dairy Science, v.74, p.483-489, 1991.

MANN, G.E.; LAMMING, G.E. Relationship between maternal endocrine environment, early embryo development and inhibition of the luteolytic mechanism in cows. Reproduction, v.121, p.175-180, 2001.

MANN, G.E.; LAMMING, G.E.; FRAY, M.D. Plasma estradiol and progesterone during early-pregnancy in the cow and the effects of treatment with buserelin. Animal Reproduction Science, v.37, p.121-131, 1995.

MANN, G.E.; LAMMING, G.E.; ROBINSON, R.S.; WATHES, D.C. The regulation of interferon-t production and uterine hormone 
receptors during early pregnancy. Journal of Reproduction and Fertility, v.54(supl.), p.317-328, 1998.

MARTINS, A.C.; MOLLO, M.R.; BASTOS, M.R. et al. Serum hormone concentrations of Zebu cows under low and high feed intake. Pesquisa Agropecuária Brasileira, v.43, p.243-247, 2008.

MATTOS, R.; STAPLES, C.R.; ARTECH, A. et al. The effects of feeding fish oil on uterine secretion of $\mathrm{PGF}_{2 \alpha}$, milk composition, and metabolic status of periparturient Holstein cows. Journal of Dairy Science, v.87, p.921-932, 2004.

MCEVOY, T.G.; ROBINSON, J.J.; AITKEN, R.F. et al. Dietary excesses of urea influence the viability and metabolism of preimplantation sheep embryos and may affect fetal growth among survivors. Animal Reproduction Science, v.47, p.7190, 1997.

MOLLO, M.R.; RUMPF, R.; MARTINS, A.C. et al. Função ovariana em novilhas Nelore submetidas a alta ou baixa ingestão alimentar. Acta Scientiae Veterinariae, v.35(Supl 3), p.958, 2007 (resumo).

MOREIRA, F.; RISCO, C.; PIRES, M.F. et al. Effect of body condition on reproductive efficiency of lactating dairy cows receiving a timed insemination. Theriogenology, v.53, p.13051319, 2000.

MURPHY, M.G.; ENRIGHT, W.J.; CROWE, M.A. et al. Effect of dietary-intake on pattern of growth of dominant follicles during the estrous-cycle in beef heifers. Journal of Reproduction and Fertility, v.92, p.333-338, 1991.

NEBEL, R.L.; JOBST, S.M.; DRANSFIELD, M.B.G. et al. Use of a radiofrequency data communication system, Heat Watch, to describe behavioral estrus in dairy cattle. Journal of Dairy Science, v.80, p.151, 1997 (resumo).

NOLAN, R.; O’CALLAGHAN, D.; DUBY, R.T. et al. The influence of short-term nutrient changes on follicle growth and embryo production following superovulation in beef heifers. Theriogenology, v.50, p.1263-1274, 1998.

O'CALLAGHAN, D.; BOLAND, M.P. Nutritional effects on ovulation, embryo development and the estabilishment of pregnancy in ruminants. Animal Reproduction Science, v.68, p.299-314, 1999.

OCON, O.M.; HANSEN, P.J. Disruption of bovine oocytes and preimplantation embryos by urea and acidic pH. Journal of Dairy Science, v.86, p.1194-1200, 2003.

OLDICK, B.S.; STAPLES, C.R.; THATCHER, W.W. et al. Abomasal infusion of glucose and fat: effect on digestion, production, and ovarian and uterine functions of cows. Journal of Dairy Science, v.80, p.1315-1328, 1997.

PARR, R.A.; DAVIS, I.F.; MILES, M.A. et al. Liver blood flow and metabolic clearance rate of progesterone in sheep. Research in Veterinary Science, v.55, p.311-316, 1993.

PERES, M.S.; SANTOS, P.G.; ANDRIGHETTO, C. et al. Efeito da semente de girassol na taxa de concepção de vacas Nelore no período pós-parto. Acta Scientiae Veterinariae, v.36, p.639, suppl.2, 2008. (Resumo).

PETIT, H.V.; CAVALIERI, F.B.; SANTOS, G.T.D. et al. Quality of embryos produced from dairy cows fed whole flaxseed. Journal of Dairy Science, v.91, p.1786-1790, 2008.

PETIT, H.V.; DEWHURST, R.J.; SCOLLAN, N.D. et al. Milk production and composition, ovarian function and prostaglandin secretion of dairy cows fed omega- 3 fats. Journal of Dairy Science, v.85, p.889-899, 2002.

PETIT, H.V.; TWAGIRAMUNGU, H. Conception rate and reproductive function of dairy cows fed different fat sources. Theriogenology, v.66, p.1316-1324, 2006.

PFEIFER, L.F.M.; SARTORI, R.; PIVATO, I. et al. Effect of circulating progesterone on in vitro developmental competence of bovine oocytes. Animal Reproduction, v.6, p.473-480, 2009.

PICCINATO, C.A.; SARTORI, R.; SANGRISTAVONG, S. et al. In vitro and in vivo analysis of fatty acid effects on metabolism of
$17 \alpha$-estradiol and progesterone in dairy cows. Journal of Dairy Science, 2010 (no prelo).

RAES, K.; DE SMET, S.; DEMEYER, D. Effect of dietary fatty acids on incorporation of long chain polyunsaturated fatty acids and conjugated linoleic acid in lamb, beef and pork meat: a review. Animal Feed Science and Technology, v.113, p.199221, 2004.

REVAH, I.; BUTLER, W.R. Prolonged dominance of follicles and reduced viability of bovine oocytes. Journal of Reproduction and Fertility, v.106, p.39-47, 1996.

RHOADS, M.L.; RHOADS, R.P.; GILBERT, R.O. et al. Detrimental effects of high plasma urea nitrogen levels on viability of embryos from lactating dairy cows. Animal Reproduction Science, v.91, p.1-10, 2006.

RHODES, F.M.; ENTWISTLE, K.W.; KINDER, J.E. Changes in ovarian function and gonadotropin secretion preceding the onset of nutritionally induced anestrus in Bos indicus heifers. Biology of Reproduction, v.55, p.1437-1443, 1996.

RHODES, F.M.; FITZPATRICK, L.A.; ENTWISTLE, K.W.; DÉATH, G. Sequential changes in ovarian follicular dynamics in Bos indicus heifers before and after nutritional anoestrous. Journal of Reproduction and Fertility, v.104, p.41-49, 1995.

ROBERSON, M.S.; WOLFE, M.W.; STUMPF, T.T. et al. Luteinizing hormone secretion and corpus luteum function in cows receiving two levels of progesterone. Biology of Reproduction, v.41, p.997-1003, 1989.

ROBINSON, R.S.; PUSHPAKUMARA, P.G.A.; CHENG, Z. et al. Effects of dietary polyunsaturated fatty acids on ovarian and uterine function in lactating dairy cows. Reproduction, v.124, p.119-131, 2002.

RYAN, D.P.; SPOON, R.A.; WILLIAMS, G.L. Ovarian follicular characteristics, embryo recovery, and embryo viability in heifers fed high-fat diets and treated with follicle-stimulation hormone. Journal of Animal Science, v.70, p.3505-3513, 1992.

SÁ FILHO, O.G.; VILELA, E.R.; GEARY, T.W. et al. Strategies to improve fertility in postpartum multiparous Bos indicus cows submitted to a fixed-time insemination protocol with gonadotropin-releasing hormone and prostaglandin F2alpha. Jounal of Animal Science, v.87, p.2806-2814, 2009.

SANGSRITAVONG, S.; COMBS, D.K.; SARTORI, R. et al. High feed intake increases blood flow and metabolism of progesterone and estradiol-17ß in dairy cattle. Journal of Dairy Science, v.85, p.2831-42, 2002.

SANTOS, J.E.P.; AMSTALDEN, M. Effects of nutrition on bovine reproduction. Arquivos da Faculdade de Veterinária da UFRGS, v.26, p.1-51, 1998.

SANTOS, J.E.P.; CERRI, R.L.A.; SARTORI, R. Nutritional management of the donor cow. Theriogenology, v.69, p.8897, 2008.

SANTOS, R.M. Efeito da quantidade de concentrado da dieta de vacas holandesas não-lactantes na progesterona plasmática, composição do fluido folicular e produção de prostaglandina pelo endométrio. 2005. Tese (Doutorado) Universidade Estadual Paulista, Jaboticabal, Jaboticabal.

SARTORI, R.; BASTOS, M.R.; MOLLO, M.R. et al. Influência da ingestão alimentar na produção de embriões bovinos. Acta Scientiae Veterinariae, v.35(Supl 3), p.869-873, 2007.

SARTORI, R.; HAUGHIAN, J.M.; SHAVER, R.D. et al. Comparison of ovarian function and circulating steroids in estrous cycles of Holstein heifers and lactating cows. Journal of Dairy Science, v.87, p.905-920, 2004a.

SARTORI, R.; LOPEZ, H.; WILTBANK, M.C. Ovarian function in the postpartum period of dairy cows. In: INTERNATIONAL CONGRESS ON ANIMAL REPRODUCTION, 15., 2004, Porto Seguro. Proceedings... Porto Seguro: 2004b. p.76-81.

SARTORI, R.; MOLLO, M.R. Influência da ingestão alimentar na fisiologia reprodutiva da fêmea bovina. Revista Brasileira de Reprodução Animal, v.31, p.197-204, 2007. 
SARTORI, R.; ROSA, G.J.M.; WILTBANK, M.C. Ovarian structures and circulating steroids in heifers and lactating cows in summer and lactating and dry cows in winter. Journal of Dairy Science, v.85, p.2813-2822, 2002a.

SARTORI, R.; SARTOR-BERGFELT, R.; MERTENS, S.A. et al. Fertilization and early embryonic development in heifers and lactating cows in summer and lactating and dry cows in winter. Journal of Dairy Science, v.85, p.2803-2812, 2002b.

SENATORE, E.M.; BUTLER, W.R.; OLTENACU, P.A. Relationships between energy balance and post-partum ovarian activity and fertility in first lactation dairy cows. Animal Science, v.62, p.17-23, 1996.

SINCLAIR, K.D.; KURAN, M.; GEBBIE, F.E. et al. Nitrogen metabolism and fertility in cattle: II. Development of oocytes recovered from heifers offered diets differing in their rate of nitrogen release in the rumen. Journal of Animal Science, v.78, p.2670-2680, 2000.

SIROIS, J.; FORTUNE, J.E. Ovarian follicular dynamics during the estrous cycle in heifers monitored by real-time ultrasonography. Biology of Reproduction, v.39, p.308-317, 1988.

SMITH, M.C.; WALLACE, J.M. Influence of early post partum ovulation on the re-establishment of pregnancy in multiparous and primiparous dairy cattle. Reproduction, Fertility and Development, v.10, p.207-216, 1998.

STAPLES, C.R.; BURKE, J.M.; THATCHER, W.W. Influence of supplemental fats on reproductive tissues and performance of lactating cows. Journal of Dairy Science, v.81, p.856-871, 1998.

STAPLES, C.R.; THATCHER, W.W.; CLARK, J.H. Relationship between ovarian activity and energy status during the early postpartum period of high producing dairy cows. Journal of Dairy Science, v.73, p.938-947, 1990.

STAPLES, C.R.; WILTBANK, M.C.; GRUMMER, R.R. et al. Effect of long chain fatty acids on lactation performance and reproductive tissues of Holstein cows. Journal of Dairy Science, v.83, p.278, 2000. (Abstract).

STEVENSON, J.S.; SCHMIDT, M.K.; CALL, E.P. Factors affecting reproductive performance of dairy cows first inseminated after five weeks postpartum. Journal of Dairy Science, v.66, p.1148-1154, 1983.

THANGAVELU, G.M.; COLAZO, G.; AMBROSE, D.J. et al. Diets enriched in unsaturated fatty acids enhance early embryonic development in lactating Holstein cows. Theriogenology, v.68, p.949-957, 2007.

THATCHER, W.W.; WILCOX, C.J. Postpartum estrus as an indicator of reproductive status in the dairy cow. Journal of Dairy Science, v.56, p.608-610, 1973.

THOMAS, M.G.; BAO, B.; WILLIAM, G.L. Dietary fats varying in their fatty acid composition differentially influence follicular growth in cows fed isoenergetic diets. Journal of Animal Science, v.75, p.2512-2519, 1997.

VELAZQUEZ, M.A.; NEWMAN, M.; CHRISTIE, M.F. et al. The usefulness of a single measurement of insulin-like growth factor1 as a predictor of embryo yield and pregnancy rates in a bovine MOET program. Theriogenology, v.64, p.1977-1994, 2005.

WATHES, D.C.; ABAYASEKARA, D.R.; AITKEN, R.J. Minireview: polyunsaturated fatty acids in male and female reproduction. Biology of Reproduction, v.77, p.190-201, 2007.

WEBB, R.; GARNSWORTHY, P.C.; GONG, J.G. et al. Control of follicular interactions and nutritional influences. Journal of Animal Science, v.82(Supl), p.E63-E74, 2004.

WESTWOOD, C.T.; LEAN, I.J.; KELLAWAY, R.C. Indications and implications for testing of milk urea in dairy cattle: a quantitative review. Part 2. Effect of dietary protein on reproductive performance. New Zealand Veterinary Journal, v.46, p.123-130, 1998.

WILLIAMS, G.L. Suckling as a regulator of postpartum rebreeding in cattle: a review. Journal of Animal Science, v.68, p.831852, 1990.

WILTBANK, M.C.; GUMEN, A.; SARTORI, R. Physiological classification of anovulatory conditions in cattle. Theriogenology, v.57, p.21-53, 2002.

WILTBANK, M.; LOPEZ, H.; SARTORI, R. et al. Changes in reproductive physiology of lactating dairy cows due to elevated steroid metabolism. Theriogenology, v.65, p.17-29, 2006.

ZERON, Y.; SKLAN, D.; ARAV, A. Effect of polyunsaturated fatty acid supplementation on biophysical parameters and chilling sensitivity of ewe oocytes. Molecular Reproduction and Development, v.61, p.271-278, 2002.

ZULU, V.C.; SAWAMUKAI, Y.; NAKADA, K. et al. Relationship among insulin-like growth factor-I, blood metabolites and postpartum ovarian function in dairy cows. Journal of the Veterinary Medicine Science, v.64, p.879-885, 2002. 